\title{
L'homme et le monde dans les philosophies anciennes
} Marie Delcourt

\section{Citer ce document / Cite this document :}

Delcourt Marie. L'homme et le monde dans les philosophies anciennes. In: Bulletin de l'Association Guillaume Budé, n³2, juillet 1931. pp. 3-10;

doi : 10.3406/bude.1931.6578

http://www.persee.fr/doc/bude_0004-5527_1931_num_32_1_6578

Document généré le 17/03/2016 


\section{L'HOMME ET LE MONDE DANS LES PHILOSOPHIES ANCIENNES}

Toutes les philosophies donnent une image du monde, les unes partant consciemment du moi, les autres inconsciemment. Toutes, dans leur étude, rencontrent l'homme à un moment donné ot le font entrer dans l'ensemble qu'elles construisent, de telle sorte qu'il y figure au moins comme objet connu, même s'il a été plus ou moins complètement éliminé comme sujet connaissant. La méthode habituelle de l'historien consiste à décrire le système que tel philosophe a construit en rectifiant pour finir la place de la planète humaine, petite terre qui voudrait se croire un pivot et qu'il a fallu débouter de son illusion. Voici un livre où est appliquée la méthode inverse : Bernard Groethuysen suppose connus les systèmes philosophiques et il se place à la périphérie de chacun d'eux pour, de là, voir l'homme. Deux questions se présentent à lui : l'une qui est une question de métaphysique : quelle relation y a-t-il entre telle image du monde et l'homme qui en est un élément? d'où vient-il et ou va-t-il ? L'autre est une question de psychologie : quel caractère a l'homme de cctte philosophie? comment se connaît-il? comment sent-il sa propre vie? comment se représente-t-il son moi et sa vie? comment imagine-t-il la construction de sa personnalité ? Il va sans dire que les deux ordres de problèmes doivent être résolus du même coup, par une description complète : la métaphysique donne les coordonnées pour situer la figure que la psychologie esquisse et qu'elle nuance 
quand elle le peut. Cette science, ou cet art, s'appelle en Allemagne l'anthropologie philosophique. Elle demande comme matériaux des textes très nombreux : nous pouvons à la rigueur nous faire une idée de la philosophie d'Héraclite, mais il nous est impossible de tracer une image plausible de ce qu'est l'homme dans un système dont nous savons si peu de chose. Même l'homme d'Epicure et de Lucrèce n'est guère pour nous plus qu'un schéma abstrait. En revanche, dans la première partie de sa Philosophische Anthropologie, parue dans le Handbuch der Philosophie de

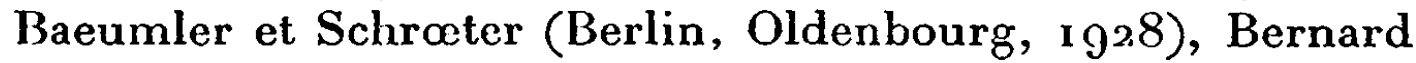
Grœthysen donne un tel relief à ce qu'est la notion d'homme chez Platon, chez Aristote, dans la philosophie latine, chez Plotin et chez saint Augustin qu'on nous permettra de résumer ici ce jpremier fascicule. Le second, qui a paru depuis, suggère d'autres problèmes.

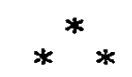

Platon pense en figures vivantes et ne peut s'imaginer un plan du monde et de la vie sans voir au centre l'homme à qui correspondent ces constructions. Socrate est l'homme pensant qui est nécessaire au monde platonicien, de même que l'univers platonicien est nécessaire à Socrate. La prodigieuse impression de réalité, de solidité que nous donne Socrate vient moins de ce qu'il a pu être de son vivant que de l'intensité avec laquelle Platon sentait le «vivre pour penser » qu'il a incorporé en Socrate. La réussite de Platon, c'est de lui avoir à la fois laissé tant de traits individuels et donné une telle signification générale.

Donner une telle primauté à l'activité pensante conduit nécessairement à accentuer l'antinomic entre l'âme et le corps. Aussi, ce que Platon cherche à situer dans le monde, ce n'est pas à proprement parler l'homme - pour cela il faut attendre Aristote - c'est l'âme, accompagnée du corps et gênée par lui. La vie ordinaire, avec ses éléments tan- 
gibles, n'a aucune réalité pour Platon. La vraie vie, pour lui, c'est la connaissance de plus en plus pénétrante, c'est une aspiration entrainant, de l'être, des éléments de plus en plus nombreux. L'âme s'harmonise et s'assainit à mesure que la fonction de connaissance y devient dominante ; elle entraîne le corps qui voudrait lui faire mener une vie pour laquelle elle n'est point faite; puis, lorsqu'elle est bien organisée, elle cherche la Justice.

Une Idée qui cesserait d'être désirée et aimée ne serait plus Idée, de même qu'une ârne qui ne chercherait plus l'Idée ne serait plus une âme. Ame et Idée ne peuvent se dissocier l'une de l'autre. Ce n'est que de leur corrélation telle qu'elle est vécue dans l'expérience philosophique que les deux termes reçoivent leur sens plein.

Quant au sort de l'homme, c'est-à-dire à l'aventure que vit l'âme dans le monde, cela, c'est de l'ordre du mythe. C'est le mythe qui rétablit l'unité entre la vie et la pensée, irréalisable ici-bas, puisque l'âme contemplatrice des Idéesn'a nullement besoin du monde, tandis que l'homme ne peut exister en dehors du monde. Le monde est nécessaire pour donner à l'homme la plénitude de son être et de sa signification : idcee qui sera développée et achevée par Aristote, tandis que le thème de l'âme sera repris dans le néo-platonisme et chez saint Augustin. Les deux thèmes conjugués détermineront toute l'ambiance philosophique du moyen âge.

Aristote est le premier à situer l'homme dans le monde et par rapport au monde, le monde étant un tout bien organisé où l'homme tient sa place. Il ne s'agit plus ici de l'homme platonicien dépouillć, transformé par l'activité philosophique, être vivant subordonné à l'âme qui pense et qui cherche. Voici l'homme normal, tel qu'il est par nature et dans la nature, déterminé par tout un ensemble de relations; il fait partie d'un système donné de moyens et de fins sans lequel il ne peut se comprendre et qui est incomplet sans lui.

La philosophie gréco-romaine - chez Gicéron, Sénèque, 
Epictète, Marc-Aurèle - reprendra cette idée, mais du point de vue de la vie individuelle. Ge qu'elle cherche à saisir, c'est la notion de la personnalité, située à l'intersection d'un caractère et d'une expérience. Il ne s'agit plus ici de définir l'homme comme élément du monde naturel et moins encore la relation de l'homme à l'éternel, mais de juger une vie humaine, c'est-à-dire une durée considérée en un individu et chargée d'événements. Dans une telle conception, il incombe à la philosophie un compte nouveau à rendre : elle accepte d'être jugée dans son efficacité à agir sur la vie, premier dialogue entre la sagesse et l'expérience, première élévation de la personnalité sur le plan philosophique. Car la personnalité véritable, c'est celle du sage qui sait exactement quel anneau il représente dans la chaîne des effets et des causes, à quelle juste place il trouvera sa liberté et son efficacité. L'homme, pour bien jouer son rôle, doit être bien portant et réaliser en soi le vœu de la nature. Ainsi, la philosophie devient un humanisme qui dépasse l'homme en cherchant à le situer dans un ensemble de relations bien établies. Quant au sage, son devoir est de se perfectionner, non dans le sens du dépouillement platonicien, mais en mûrissant ce qui, dans son individualité, sert le mieux les fins auxquelles il est, comme individu, subordonné. Il est indispensable qu'il se voie lui-même tel qu'il est dans le monde, ni trop petit ni trop grand. Lorsqu'il aura pris cette exacte conscience de soi, il constatera les obstacles et il les vaincra. Sagesse et volonté sont les deux aspects de la personnalité : agir en accord avec soi-même et avec la nature. L'ètre n'est qu'en partie donné à la naissance : c'est la volonté personnelle qui achève de le construire et de l'harmoniser avec le tout. L'affirmation des philosophes latins, ce n'est plus : « je suis », mais « je suis moi ». La connaissance pure cède devant le primat de la vie.

Toute opposéc est la position de Plotin. L'homme sait que son âme n'est qu'unc fraction d'unc âme immense qui dépasse et déborde de chaque moi individuel. Le mythe, qui 
chez Platon complète simplement le tableau du monde, envahit ici tout le champ. Le monde mythique devient un tout organisé, auquel l'homme philosophant, cherchant et aspirant ne s'oppose plus, parce que l'homme lui-même est devenu un élément de ce mythe. "La conception mythique se transforme en un événement mystique, d'ou aucune route ne ramène plus à la vie humaine (p. 77) ». Personnalité dans le sens latin signifie possession de soi. L'âme humaine instruite par Plotin s'évade au contraire pour s'en aller, par delà l'Ame du monde, retrouver l'Un et s'unir à lui en une fusion d'où elle souhaite de ne jamais plus revenir vers la simple humanité.

Augustin renoue avec la tradition latine, non sans de grandes différences. Etonnement devant soi-même comme unité vivante, admiration, effroi devant l'âme, sentiment que la vie est une chose incommensurable, insaisissable. Entre le temps qui coulait avant nous et qui coulera après nous, d'une part, et d'autre part nous-mêmes qui aspirons non à une durée mille fois plus longue que notre aevum, mais à l'éternel, il n'y a pas de comparaison possible. L'homme devient un vouloir tout tendu vers le transcendant, en lutte active avec tout ce qui empêche l'ascension. La philosophie latine dit: «bien s'acquitter de la vie », Augustin; dit “dominer la vie» (p. q I). “En face des tendances néoplatoniciennes, l'augustinianisme est une méthode pour ramener l'homme à lui-même. L'âme cosmique redevient un élément de l'homme " ( $\left.p .9^{2}\right)$.

De plus, chaque homme pour son propre compte devient protagoniste dans le drame du péché. "Parce qu'il est le pécheur, il est la seule figure tragique en ce monde 》 (p. 87 ). Lui seul pose un problème dans un monde qui n'en comporte pas. L'expérience d'Augustin lui-même est l'expérience typique de l'humanité pécheresse, mais redressée par le besoin de l'éternel; et c'est pour cela qu'Augustin a écrit ses confessions. Mais le drame recommence avec des données nouvelles pour chaque individu et l'on ne dit plus «l'âme » 
mais « mon âme ». Quant à Dieu, il est, comme l'Idée dans le système platonicien, une notion corrélative à celle d'homme : ce n'est pas le Dieu en soi, mais le Dieu de l'homme, à qui l'homme dit « mon Dieu ». Ce n'est pas davantage l'homme en soi, mais l'homme qui a péché envers Dieu et qui lui demande rédemption. Disons tout de suite qu'il est fort douteux que des théologiens acceptent ces deux dernières propositions, car Dieu aurait parfaitement pu ne pas créer l'homme, dont l'apparition sur la terre est toujours présentée comme une preuve parfaitement gratuite de la bonté de Dieu. Quoi qu'il en soit, tous les élćments de l'anthropologie antique se retrouvent chez Augustin, réordonnés d'après le plan chrétien.

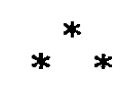

Après ce court résumé, indiquons le seul point important où il nous paraît impossible d'accepter l'interprétation de Groethuysen. Celui-ci appartient à l'école philosophique allemande d'après Nietzsche, dont tous les représentants s'accordent pour attacher beaucoup d'importance aux méthodes et presque aucune aux doctrines. Groethuysen, qui est leur historien - on connaît son Introduction à la pensée philosophique allemande depuis Nietzsche ${ }^{1}$ - l'a dit à propos de chacun d'eux. Nietzsche estime «qu'une philosophie ne saurait se concevoir qu'en fonction d'un philosophe. Celui-ci, sans le savoir et sans le vouloir, écrit à sa manière ses mémoires » (Introduction, p. 17). “ Ainsi disparaît l'illusion de la connaissance pure qui fut longtemps l'idole du philosophe »(p. 22). Dilthey, après avoir réfuté tous les systèmes métaphysiques, dit : "La métaphysique en tant qu'elle prétend être une science, ne représente qu'une manifestation de l'esprit limitée à certaines époques, mais la conscience métaphysique de l'individu est éternelle» (p. 50). Simmel

I. Paris, Stock, 1926 . 
" aura plutôt formé un esprit philosophique que développé une philosophie, car on peut voir les choses en métaphysicien sans adopter, à proprement parler, une métaphysique »(p. 85). Cela est parfaitement défendable, mais aucun état d'esprit n'est plus étranger aux philosophes de l'antiquité. Dès lors, peut-on dire, comme fait Groethuysen, que le dialogue est pour Platon, non une méthode d'exposé, mais l'essence mème du " philosopher » platonicien, l'expression naturelle et nécessaire du mouvement de l'esprit qui monte à la découverte de sa vérité ? que la philosophie platonicienne est un climat, le climat même de la philosophie, plutôt qu'un système, un enseignement, une conception du monde ? En réalité, Platon s'est fait illusion sur la valeur démonstrative de la forme dialoguée. Groethuysen a certes raison de montrer qu'elle est une expression excellente pour l'homme, Socrate s'adressant à des hommes, agissant sur eux, stimulé par eux, composant avec eux l'équipe qui s'en va, guidc et voyageurs, à l'ascension de la vérité. Mais, si Platon l'a choisie, c'est avant tout qu'il y voyait la traduction d'une dialectique capable d'acquérir des vérités éternelles. Car la vérité existe, et l'intelligence de l'homme est habile à la découvrir, puisque l'intelligence et le monde sont accordés aux mèmes lois. L'exposć du Timée situe l'homme dans le monde - l'homme et non seulement l'âme humaine - aussi clairement que la philosophie aristotélicienne.

Cela nous amène à dire un mot du mythe dans Platon, auguel il nous parait impossible de donner la signification que veut Groethuysen. Pour lui, chez Platon et à plus forte raison chez. Plotin, le mythe est toujours une évasion dans l'irrationnel. Et il est certain qu'il y a des mythes platoniciens qui sont cela : le philosophe, sentant inconnaissables certaines réalités, les remplace par une image sur le caractère provisoire de laquelle il ne se méprend nullement, ni ses lecteurs. Mais, dans bien d'autres cas, l'imagination vient simplement - ou croit venir - au secours de la raison, étoffant de représentations sensibles une donnée rationnelle- 
ment démontrable, ou encore narrant la naissance dans le temps d'une réalité éternelle. Ici encore, Platon s'est fait illusion, cette fois sur la valeur de l'allégorie comme adjuvant de la connaissance. De même que la forme dialoguée, la forme mythique crée pour nous des quantités de problèmes autour du problème central. Platon écrivait pour un peuple qui traduisait spontanément en mythes les réalités spirituelles dont il vivait. Il n'a pas prévu qu'il serait lu par des hommes à qui cette méthode intellectuelle serait devenue totalement étrangère. Parce que le mythe se développe pour nous au delà de la frontière qui termine nettement le domaine de la raison, ce serait certainement une erreur de ne considérer comme doctrine, chez Platon, que ce qui est en deçà de la zone mythique.

Marie Delcourt. 